\title{
Forum
}

\section{Par-delà le visible}

\author{
Claudine Friedberg \\ Anthropologue, Département Hommes, Natures, Sociétés, Muséum national d’histoire naturelle, Bâtiment 135, 57 rue Cuvier,
} 75231 Paris cedex 05, France

Mots-clés : ontologies ; nature ; sociétés; comparatisme ; animisme

\section{Keywords:}

ontologies;

nature;

societies;

comparatism;

animism

\begin{abstract}
Résumé - Les relations aux êtres qui peuplent les milieux de vie des différentes sociétés humaines ont évolué et continuent à évoluer dans le temps et l'espace. Un ouvrage comme celui de Philippe Descola (Par-delà nature et culture), qui a pour objectif de mettre de l'ordre dans ces relations en repérant sur quels schèmes de pratiques elles sont fondées, ne peut laisser indifférents tous ceux qui s'intéressent à l'environnement aujourd'hui. Son analyse le conduit, en partant des modes d'identification de «l'autre ", à construire quatre ontologies (animisme, totémisme, naturalisme et analogisme), permettant de classer les sociétés humaines en fonction de leur façon d'envisager les autres existants et les rapports qu'elles entretiennent avec eux. Cette entreprise ne manque pas de susciter des critiques quant au résultat, surtout de la part d'anthropologues qui ne reconnaissent pas dans les sociétés qu'ils ont étudiées les caractéristiques proposées par l'auteur. Il était donc nécessaire de suivre pas à pas ses raisonnements avant de voir en quoi un tel ouvrage peut être critiqué, en quoi il peut apporter des pistes de réflexion.
\end{abstract}

\begin{abstract}
Beyond Nature and Culture. Relationships with all the other beings that inhabit the living environments of human societies have evolved over time and continue to do so. A book such as Philippe Descola's ("Beyond Nature and Culture"), which aims to order these relationships by identifying the underlying schemes of practices, is of interest to all people dealing with the environment today. Starting from the ways "the other" is identified, his analysis leads him to develop four ontologies (animism, totemism, naturalism and analogism). These enable him to classify human societies according to the way they consider other non-human beings and to the relationships they entertain with them. This endeavour has not failed to generate criticism, mainly from anthropologists who do not recognize the features proposed by the author in the societies they have investigated. Therefore, we needed to follow his reasoning step by step in order to understand the arguments which are to be questioned or adhered to. We consider first the relevance of the division into four ontologies. We then criticize some aspects of the author's approach and especially the way he avoids dealing with the dynamics that have affected human societies over time, especially in their relationships with animals and plants.
\end{abstract}

Au moment où la majeure partie de l'humanité bascule dans un mode de vie urbain, on s'interroge de plus en plus sur nos relations aux autres êtres vivants et aux différents éléments naturels de notre environnement, alors même que ces relations cessent de s'inscrire, pour la plupart d'entre nous, dans des pratiques quotidiennes. Dans le même temps, nombre d'écrits revendiquent la nécessité d'envisager autrement le regard que nous portons sur ce que nous avons été habitués à appeler la «nature». Ce sentiment atteint le grand public à travers des textes qui le vulgarisent de façon plus ou moins légitime. On parle de «nouvelle alliance » à la suite de Jacques Monod et de Prigogine, de «contrat naturel » avec Michel Serres, de Gaia, un système-terre autorégulé, avec James Lovelock, de la montagne qui pense avec Aldo Leopold, tandis qu'avec la vague New Age, on s'extasie sur la prétendue harmonie dans laquelle vivraient les peuples indigènes, en empruntant aux anthropologues des données fragmentaires isolées de leur contexte.

Il ne faut donc pas s'étonner de l'écho rencontré par l'ouvrage Par-delà nature et culture ${ }^{1}$, dans lequel Philippe Descola se propose de répertorier et d'ordonner les diverses modalités selon lesquelles les membres des différentes sociétés humaines ont envisagé et pratiqué leurs relations aux autres « existants ». Il déploie pour cela une grande érudition, non seulement pour ce qui est des

Auteur correspondant : friedbg@mnhn.fr

1 Descola, P., 2005. Par-delà nature et culture, Paris, Gallimard. 
données anthropologiques et en particulier des controverses récentes à propos du concept d'animisme, mais aussi en ce qui concerne l'histoire des idées en Occident.

On peut cependant se demander si cet ouvrage répond pleinement au désir qui se manifeste actuellement de mieux comprendre notre rapport au monde. Sa qualité première est de balayer les idées trop simplistes qui ont pu surgir ici ou là sur les relations que les sociétés humaines entretiennent ou ont entretenu avec les êtres qui peuplent leur environnement. Il n'en demeure pas moins qu'il est difficile d'accepter certains aspects des élaborations théoriques exposées par Descola et, en même temps, de les critiquer, tant il prend de précautions en les énonçant, allant jusqu'à fournir lui-même les arguments qui pourraient les contredire.

Je développerai quelques-unes des questions que soulève cet ouvrage, en particulier pour les anthropologues du fait que leurs données s'intègrent souvent mal dans le découpage proposé par l'auteur. Je m'interrogerai aussi sur la façon dont ce dernier évite de traiter des dynamiques qui ont affecté les sociétés humaines au cours des temps. Mais auparavant, pour permettre au lecteur de comprendre mon propos, il me faut expliciter le contenu du livre, selon mon point de vue, et en offrir une lecture synthétique. Cela me permettra d'évoquer la richesse des données fournies et d'amorcer l'analyse critique des interprétations développées par Descola.

\section{L'opposition nature/culture}

Dans une première partie, l'auteur montre que la coupure nature/culture que nous connaissons en Occident est inconnue dans d'autres sociétés. Pour cela, il part de sa propre expérience de terrain chez les Achuar, en Amazonie équatorienne, dont la cosmologie ne fait pas de discrimination entre humains et non humains, mais différencie les êtres en fonction de leur mode de communication et de leur type d'âme. Puis il montre que ce cas n'est pas isolé et comment, pour d'autres populations à travers le monde et vivant dans des milieux différents, l'environnement n'est pas objectivé comme une sphère autonome. Certes, cette idée n'est pas nouvelle, mais elle va à l'encontre de ce que les anthropologues ont pu longtemps défendre, en particulier à la suite des travaux de Lévi-Strauss.

Descola explore ensuite les différentes formes qu'aurait pu prendre cette coupure : opposition entre sauvage et domestique ou différenciation entre des espaces plus ou moins humanisés. Il conclut que, aussi bien chez des chasseurs-cueilleurs que chez des agriculteurs, plus ou moins sédentarisés, une telle dichotomie n'existe pas. Pour les Achuar, elle renvoie à une opposition entre "cultivé par les esprits » et "cultivé par les hommes». La façon dont ils se comportent en forêt montre combien cet espace leur est familier. Il en est de même chez d'autres Amérindiens amazoniens, conscients que le déplacement de leurs jardins éphémères aboutit progressivement à une humanisation de l'espace forestier, ce que confirment les travaux en écologie tropicale.

Après avoir évoqué l'histoire de l'opposition entre la «sauvagerie » et le «cultivé » en Occident, Descola remarque qu'en Chine, en Inde ou au Japon, une opposition comparable est difficilement repérable tant ces lieux sont investis «de valeurs qui ne sont pas mutuellement exclusives ». Il examine ensuite comment s'est effectué en Occident le «grand partage » entre nature et culture, en commençant par une réflexion sur l'autonomisation du paysage dans la peinture. Puis il suit ce processus depuis ses débuts en Grèce et «l'autonomie de la Création » qui, avec le christianisme, héritier de la tradition juive, fait de l'homme un être extérieur et supérieur à la nature. Avec la révolution scientifique, cette dernière s'autonomise au début du XVII siècle. L'auteur suit ainsi l'évolution des idées jusqu'à l'avènement des concepts de société, de culture, et celui de civilisation. Pour lui, l'anthropologie est le fruit de cette longue maturation, en particulier à travers l'œuvre de Durkheim.

\section{Les quatre modes d'identification et les quatre ontologies}

Descola développe ensuite sa théorie des quatre ontologies, qui est l'hypothèse originale développée dans ce livre. Il annonce clairement ses intentions (p. 144) : «Ce livre repose sur le pari qu'il est possible de mettre au jour des schèmes élémentaires de la pratique et de dresser une cartographie sommaire de leur distribution et de leurs arrangements. » Il dit plus loin (p. 151) que « ces schèmes collectifs peuvent être soit non réflexifs » - ils n'affleurent donc pas à la conscience -, «soit explicitables ». Il se réfère alors aux acquis des travaux sur la cognition humaine qui nous permettent maintenant de mieux comprendre comment des pratiques se mettent en place sans affleurer à la conscience, comment procède l'apprentissage des savoir-faire et l'importance des aspects non linguistiques de celui-ci.

Pour illustrer ce qu'il entend par schèmes de la pratique, Descola donne en exemple la fameuse correspondance établie par Haudricourt entre le traitement d'autrui et les pratiques à l'égard des animaux et des plantes dans l'agriculture : action directe positive vis-à-vis de ses moutons pour le berger méditerranéen; action indirecte négative dans la culture de l'igname en Mélanésie. La transmission et la perpétuation des schèmes s'effectuent, dit-il, à travers leur actualisation dans les rituels et le rôle joué par l'émotion lors de ces événements remarquables.

Dans un chapitre intitulé «Rapport à soi, rapport à l'autre ", il en arrive à l'hypothèse qui sert de fil conducteur aux développements qui vont suivre : «[...] les 
schèmes intégrateurs des pratiques [...] peuvent être ramenés à deux modalités fondamentales de structuration de l'expérience individuelle et collective : l'identification et la relation» (p. 163). «[...] l'identification - écrit Descola - est le schème au moyen duquel j'établis des différences et des ressemblances entre moi et des existants en inférant des analogies et des contrastes. » Il affirme ensuite que, sur le plan logique, il y a antériorité et donc extériorité de l'identification par rapport à la relation. Il admet pourtant qu'il s'agit «d'un mécanisme de médiation entre le soi et le non-soi », ce qui implique déjà, me semble-t-il, une relation.

Il en vient alors à la distinction qui va lui permettre de définir quatre ontologies fondées sur quatre modes d'identification : son hypothèse est que, partout dans le monde, on distingue pour tout être une intériorité et une physicalité analogues ou non à celles que nous nous attribuons à nous-mêmes.

Qu'est-ce que Descola désigne exactement par ces termes? Il se défend d'abord de vouloir revenir à l'opposition occidentale entre le corps et l'esprit, et pourtant, comme on peut le voir dans la suite de l'ouvrage, cela y ressemble bien souvent. Mais examinons de plus près ses définitions. Intériorité (p.168) : «gamme de propriétés reconnues par tous les humains et recouvrant en partie ce que nous appelons d'ordinaire l'esprit, l'âme ou la conscience - intentionnalité, subjectivité, réflexivité. Il s'agit d'une croyance universelle qu'il existe des caractéristiques internes à l'être [...]. Par contraste, la physicalité concerne la forme extérieure, la substance, les processus physiologiques », mais aussi «le tempérament, la façon d'agir dans le monde ».

Il discute ensuite de l'universalité du sentiment de soi comme singularité autonome. Il appuie sa conviction sur le fait qu'il existerait dans toutes les langues des moyens d'exprimer le «je » et le «tu». Il admet pourtant que des anthropologues ont mis en évidence, dans maintes sociétés, que la personne dépend d'éléments qui lui sont extérieurs ${ }^{2}$.

Évoquant tout ce qui pourrait contredire ou affaiblir ses hypothèses, il déclare que la conscience de soi « mêle de façon inextricable le sens d'une unité interne [...] et l'expérience $d^{\prime}$ un corps [...] source de sensation propres, organe d'une médiation avec l'environnement [...]. [...] chacun sait que l'on pense aussi avec le corps » (p. 171).

Il prend en compte différentes formes attribuées à l'intériorité, par exemple l'existence d'âmes multiples (17 chez les Indiens Tzeltal, 8 chez les Dogons) pouvant avoir pour siège certaines composantes du corps. Il convient que, selon les populations, il existe différentes

\footnotetext{
${ }^{2}$ Descola cite à ce propos les travaux de M. Strathern, en Mélanésie, qui a proposé de parler de la personne non comme une individualité, mais comme une "dividualité », "c'est-àdire un être défini [...] par sa position et ses relations dans un réseau» (p.170).
}

Tableau. Les quatre ontologies d'après Descola (2005, p. 176).

\begin{tabular}{|c|c|c|}
\cline { 2 - 3 } \multicolumn{1}{c|}{} & $\begin{array}{c}\text { Ressemblance des } \\
\text { physicalités }\end{array}$ & $\begin{array}{c}\text { Différence des } \\
\text { physicalités }\end{array}$ \\
\hline $\begin{array}{c}\text { Ressemblance des } \\
\text { intériorités }\end{array}$ & Totémisme & Animisme \\
\hline $\begin{array}{c}\text { Différence des } \\
\text { intériorités }\end{array}$ & Naturalisme & Analogisme \\
\hline
\end{tabular}

formes de connexions entre intériorité et physicalité, mais maintient qu'il y a partout une distinction entre les deux et que cette dualité n'est pas propre à l'Occident. Cette opposition telle qu'elle s'est forgée en Europe ne serait qu'une variante locale de ce qu'il présente comme une aptitude innée.

On en arrive enfin aux quatre ontologies définies par les quatre combinaisons possibles dans les modes d'identification, selon que l'intériorité et/ou la physicalité sont considérées comme semblables ou différentes, ainsi que le montre le tableau ci-dessus. Il les désigne par des termes (animique, totémique, naturaliste, analogique) auxquels il donne une acception nouvelle, même par rapport avec ce qu'il avait pu écrire lui-même auparavant. En effet, il explique qu'il abandonne sa précédente définition de l'animisme comme correspondance entre le traitement social des humains et celui des plantes et des animaux, parce qu'elle réintroduisait un dualisme entre nature et sociétés. Il déclare : «Il fallait donc se déprendre du préjugé sociocentrique ${ }^{3}$ et faire le pari que les réalités sociologiques - les systèmes relationnels stabilisés - sont analytiquement subordonnées aux réalités ontologiques - les systèmes de propriétés imputées aux existants $\gg($ p. 180).

\section{Les quatre ontologies et la façon d'appréhender les non-humains}

Descola se livre alors à un examen détaillé des quatre ontologies, en commençant par celle qu'il connaît le mieux au travers de sa propre expérience : l'animisme. Cependant, les exemples présentés ne sont pas pris uniquement en Amazonie, mais aussi en Amérique du Nord, en Malaisie et en Mélanésie.

On est alors confronté à la difficulté de cerner « ce » qui est physicalité et de la différencier de l'intériorité. La première "réside dans la forme mais aussi le mode vie qu'elle induit » (p. 184), alors que la substance fait partie de «ce » qui est intériorité. Dans l'ontologie animique, les organismes ne se distinguent que par leur enveloppe. Pourvus d'une intériorité identique, les êtres ont donc la

\footnotetext{
${ }^{3}$ Descola signale à plusieurs reprises qu'il s'agit là de l'héritage de Durkheim.
} 
capacité de se métamorphoser et d'apparaître sous des aspects différents.

Descola examine la façon dont les populations animistes considèrent la vision que les non-humains ont d'eux-mêmes. Par exemple, pour certaines populations, le jaguar et le pécari se perçoivent comme des humains. Les populations amazoniennes ainsi que certaines populations d'Amérique du Nord, par exemple les Sioux, pensent que les non-humains vivent selon le même modèle social et culturel que les humains. Les choses se compliquent avec l'examen de ce que Descola appelle le «perspectivisme» à la suite de Viveiros de Castro, où l'on se préoccupe du point de vue des non-humains sur les humains. Le lecteur est alors confronté à un abîme de mise en perspective dans lequel on ne sait plus trop comment est identifié l'autre et par conséquent le soi-même.

Avec sa seconde ontologie (ressemblance des physicalités et des intériorités), Descola reprend la question du totémisme tout en refusant de le considérer, à l'instar de Lévi-Strauss, comme une méthode classificatoire établissant une « homologie des écarts différentiels entre une série naturelle, les espèces éponymes, et une série culturelle, les segments sociaux » (p. 204).

Bien que le terme totem ait été emprunté aux Ojibwa en Amérique du Nord, ce sont les aborigènes australiens - sur lesquels on a eu des données dès la fin du XIX ${ }^{e}$ siècle - qui ont servi de terrain privilégié pour appréhender le totémisme. En dépit d'une grande diversification, les faits australiens ont une coloration particulière due à un enracinement dans une cosmologie qui se rapporte à ce qu'il est convenu d'appeler le temps du "rêve », celui des origines et de la mise en forme du monde. Des êtres sortirent de terre en des lieux précis puis disparurent, mais ils laissèrent en place les humains et les non-humains qui peuplèrent l'Australie, déjà répartis en groupes totémiques. Dans les lieux où ils disparurent, ils abandonnèrent des stocks d'esprits destinés à s'incorporer dans des individus ayant cette espèce ou objet pour totem. Ainsi s'enclenche un mouvement de génération continue où se trouvent associés des humains et des non-humains, les uns et les autres partageant avec leur totem certaines propriétés, et une identité de nature entre eux consistant en un ensemble d'attributs moraux, physiques et comportementaux.

Descola revient brièvement aux totems algonkins pour constater que certains de ces groupes amérindiens relèvent de l'animisme associé avec du totémisme. Il dit alors, et le répétera plusieurs fois au cours de l'ouvrage, qu'il peut y avoir coexistence entre les quatre modes d'identification, mais que l'un toujours prédomine.

Avec sa troisième ontologie (ressemblance des physicalités, différence des intériorités), il en arrive au mode de pensée propre à l'Occident moderne, le naturalisme, formule inversée de l'animisme : les humains se différencient des non-humains par «la conscience réflexive, la subjectivité, le pouvoir de signifier, la maîtrise des symboles et le langage au moyen duquel ces facultés s'expriment» (p. 243). En revanche, nos composantes physiques nous situent dans un continuum en raison de notre héritage phylogénique, depuis notre structure moléculaire jusqu'au fonctionnement de notre métabolisme.

Il fait ensuite une recension des différentes contestations qui ont pu s'exprimer quant aux capacités prêtées aux animaux par rapport à celles de l'homme depuis Montaigne (Descartes, Condillac...) et se demande si, avec les dernières observations des éthologistes comportementaux, un verrou du naturalisme n'aurait pas sauté.

Puis il examine les courants de pensée contemporains qui tendent à éliminer l'existence même de l'esprit. D'abord ceux qui considèrent la cognition comme une rétroaction continue entre un organisme et le milieu ambiant. Son aspect le plus radical est la théorie écologique de la vision, de James Gibson, qui élimine totalement l'esprit et dans laquelle l'action n'est plus qu'une réaction et un ajustement permanents à ce que l'on perçoit des conditions environnementales. Un autre courant nourri des recherches récentes en neurosciences remet en question l'autonomie de l'intériorité humaine et considère la pensée comme le produit de l'activité du cerveau. Les possibilités fournies par l'IRM (imagerie par résonance magnétique) de "voir » cette activité conduisent ainsi à l'abandon de l'existence d'une intériorité immatérielle. Mais, ajoute Descola, il reste des « résidus » non visibles, comme la conscience de soi, et il conclut en disant que «l'esprit a encore de beaux jours devant lui » (p. 267-268).

À ses yeux, c'est dans le domaine de la philosophie morale et du droit que l'ontologie naturaliste « encourt les risques d'effritement les plus sérieux » avec l'apparition des «droits de la nature ». Il évoque « l'émergence d'une approche proprement morale des devoirs de l'homme vis-à-vis de la collectivité du vivant et des droits que celle-ci pourrait posséder de façon intrinsèque » (p. 268) dans certains pays, dont les États-Unis, 1'Australie, 1'Allemagne et les pays scandinaves, les nations latines restant à l'écart de ce mouvement. Il y voit un courant de pensée lié pour les uns aux valeurs du protestantisme, pour l'Allemagne à la permanence de certains types de rapports au sauvage. Quant à l'Australie et aux ÉtatsUnis, il pense que cette attitude passe par une mauvaise conscience vis-à-vis des autochtones. Toutefois, ces divers mouvements ne remettent pas en cause, selon lui, les fondements du naturalisme puisqu'ils reconnaissent toujours "continuité des corps, d'un côté, discontinuités des facultés mentales de l'autre » (p. 271), même si certains reconnaissent la qualité de sujet à des primates proches de l'homme, comme le chimpanzé.

Pour la quatrième et dernière ontologie, l'analogique (différence des physicalités et des intériorités), c'est la majeure partie de l'humanité que l'on aborde puisque l'on 
y trouve, outre l'Occident jusqu'aux temps modernes, l'Inde, la Chine, l'Amérique des grandes civilisations du Mexique au Pérou, diverses sociétés andines, la majorité des sociétés de 1'Asie du Sud-Est, de la Mélanésie et de l'Océanie.

L'analyse commence par l'histoire de l'ontologie analogique, qui a régné de façon presque hégémonique sur la culture occidentale depuis l'Antiquité et au moins jusqu'à la Renaissance. Cette ontologie s'accompagne d'une croyance dans une chaîne des êtres qui se répartissent selon leur degré de perfection - l'homme étant au sommet - et d'une correspondance entre microcosme et macrocosme.

Descola s'étend peu sur les manifestations de cette ontologie en Chine pour s'attarder plus longuement sur les formes qu'elle a prises au Mexique où, selon les populations, les composantes de la personne sont localisées dans certains organes qui commandent en même temps les fonctions physiologiques et psychiques ainsi que les affects, rendant problématique leur répartition entre intériorité et physicalité.

Il évoque alors pour la première fois les incompatibilités entre systèmes ontologiques : il oppose ainsi la prise de drogues dans les systèmes animiques, destinée à « délivrer l'intériorité de son carcan physique », et le voyage volontaire vers le monde des esprits des chamanes amérindiens ou sibériens, aux phénomènes de possession qu'il dit propres aux ontologies analogiques. Il souligne aussi la place prépondérante des ancêtres et des rituels sacrificiels, qui serait caractéristique des ontologies analogiques et inconnue dans les systèmes animiques ${ }^{4}$.

Dans ce contexte, Descola prend également position par rapport aux travaux de Louis Dumont. Pour lui, ce que ce dernier appelle holisme, « à savoir un système de valeur qui subordonne la place de chaque existant [...] à une totalité transcendante », ne serait pas la caractéristique de l'ensemble des sociétés non modernes, " mais plutôt un moyen employé par les seules ontologies analogiques afin de rendre maîtrisable un amoncellement de singularités » (p. 316).

Ses réflexions portent ensuite sur l'articulation du continu et du discontinu dans les différentes ontologies et sur la manière dont elles diffèrent $\mathrm{du}$ point de vue des processus classificatoires. Il distingue l'identification par attributs qui, d'après lui, serait le propre du naturalisme et de l'analogisme, et l'identification par référence à un prototype que l'on trouverait dans l'animisme et, sous une autre forme, dans le totémisme. Les réflexions de Descola sur les processus classificatoires sont

\footnotetext{
4 Pour le sacrifice, il s'appuie sur la théorie de Lévi-Strauss qui le désigne comme un moyen « $\mathrm{d}$ 'instaurer un rapport de contiguïté » (p. 318) entre des termes entre lesquels il n'existe aucun lien au départ, pour constater que cette médiation serait inutile et incongrue dans le cas de l'animisme où la victime sacrificielle possède une intériorité semblable à celle des humains.
}

trop succinctes pour en discuter de façon pertinente. Je tiens simplement à signaler que les deux types d'identification qu'il reconnaît, prototypique ou par attributs, sont le plus souvent utilisés conjointement par les mêmes populations.

Descola aborde ensuite la question des " collectifs " correspondant à chacune des quatre ontologies. Il redit alors que, dans l'animisme, les non-humains dotés d'une intériorité analogue à la nôtre "sont réputés vivre au sein de collectifs possédant une structure et des propriétés identiques » (p. 342) à ceux des humains. Il précise dans une note que le terme " collectif » est pris ici au sens de Latour, c'est-à-dire un rassemblement d'humains et de non-humains, ce qui distingue cette notion de celle de société. Cependant, il reprendra plus loin ce concept de «collectifs » en disant que les limites de chacun d'eux sont définies par la prévalence d'un schème de relations spécifiques et déclare alors se démarquer de la définition de Latour. Ces limites ne correspondent pas au découpage habituel en ethnies, tribus, groupes linguistiques, découpage d'ailleurs actuellement contesté en anthropologie.

Il s'interroge enfin sur le concept de «sujet » et sur la définition de l'altérité dans les différentes ontologies.

\section{Écologie des relations}

Ce n'est que dans la dernière partie de l'ouvrage que Descola s'attaque de front aux "modes de relations qui viennent moduler chaque mode d'identification » (p. 425). Il répartit en deux groupes les relations dont il décide de traiter, tout en admettant qu'il en existe d'autres. Le premier est caractérisé par des relations potentiellement réversibles : ce sont l'échange, la prédation et le don. L'autre concerne « des relations univoques fondées sur la connexité » (p. 425) : ce sont la production, la protection et la transmission.

Les relations du premier groupe, qui correspondent aux actions : donner, prendre, échanger, ont une longue histoire dans la littérature anthropologique, comme le rappelle Descola. Citons, entre autres, le fait que LéviStrauss ait accordé un rôle crucial à l'échange, en particulier pour justifier la prohibition de l'inceste, mais aussi, évidemment, le fameux Essai sur le don de Marcel Mauss, sur lequel les anthropologues continuent de gloser.

Descola, pour sa part, tient à distinguer le don, qui n'oblige pas forcément à la réciprocité, de l'échange, qui implique une contrepartie. Il évoque les travaux qui font de l'échange et du don la caractéristique des chasseurscueilleurs et en particulier ceux de Bird-David, qui a développé la conception «d'environnement donateur » (p. 433) dans les rapports à la forêt. Il cite aussi Ingold, pour qui les relations de «partage» sont une manifestation du concept de «confiance» (p. 434), donc une 
situation de dépendance librement consentie. Le partage est également valorisé dans les sociétés amazoniennes qui pratiquent aussi l'agriculture.

Il revient ensuite sur la prédation, «mécanisme central de la préservation du vivant» (p.435), mais que « certains collectifs » ont adoptée comme «moyen paradoxal d'incorporer l'altérité ». Il s'agit d'une « relation aux humains et aux non-humains fondée sur la captation de principes d'identité et de substances vitales réputées nécessaires à la perpétuation de soi » (p. 436). C'est ce qu'il a pu observer chez les Achuar et d'autres sociétés amazoniennes. Il en conclut que « la philosophie de l'échange paritaire » (p. 437) ne peut donc les caractériser de façon exclusive.

Il en arrive alors (p. 439) au deuxième groupe de relations. L'idée de production, dit-il, n'est « guère adéquate pour définir la manière dont les chasseurs-cueilleurs conçoivent leurs techniques de subsistance» (p. 441), mais elle ne l'est pas davantage pour qualifier, dans la civilisation chinoise, la conceptualisation $\mathrm{du}$ processus d'engendrement des choses.

La protection implique une domination non réversible et devient un schème dominant lorsque des plantes et des animaux sont perçus comme tributaires de l'action des humains dans le cadre de la domestication.

Quant à la transmission, elle est ce qui permet l'emprise des morts sur les vivants, qui se manifeste par " une véritable dette des vivants à l'égard des morts " (p.450), phénomène particulièrement flagrant en Afrique de l'Ouest. D'ailleurs, il est significatif, dit-il, que ceux qui contestent le primat de l'ancestralité, comme Ingold, prennent leurs exemples dans les collectifs animiques où les morts sont exclus des collectifs humains.

Pour illustrer les combinaisons résultant de la conjonction entre un mode d'identification et un mode de relation, il se borne à l'animisme en raison de la grande diversité des variantes qu'il offre. Il reprend l'exemple des Achuar pour illustrer "l'appropriation belliqueuse d'autrui. Puis, en contre-exemple, il traite des Tukano de Colombie vivant dans le même milieu, et plus particulièrement des Desana et de leur modèle thermodynamique où l'énergie du Cosmos fournie par le Père Soleil est finie et où l'important est d'assurer une bonne circulation de celle-ci avec échange d'âmes humaines contre des animaux à chasser, échange négocié à l'avance par les chamanes. Il ajoute que la même attention à une réciprocité équilibrée, en particulier avec le gibier, se retrouve en Guyane, dans les forêts sibériennes et chez les Moî du Viêt Nam.

Descola présente enfin un autre exemple d'animistes amazoniens : les Campas du Pérou, chez lesquels les animaux se donnent par "pure bienveillance », créant ainsi «des obligations morales aux chasseurs » (p. 483).

Chez les Algonkin et les Inuit, c'est aussi par un sentiment de générosité que le gibier fait don de son enveloppe charnelle au chasseur; son âme se réincorpore dans un individu de la même espèce à condition que les rituels appropriés aient été effectués.

Dans le dernier chapitre, "Histoires de structures", Descola semble s'engager, comme le titre l'indique, dans la diachronie. Ainsi, après avoir constaté que «les cultures et les civilisations font preuve d'une remarquable permanence du point de vue de leur vision du monde » (p. 497) et qu'il est difficile de repérer les ruptures structurelles, il s'interroge sur la façon dont « un mode de traitement d'autrui devient caduc et un autre jusque-là marginal devient dominant », ouvrant ainsi « la voie à des innovations techniques » (p. 498).

Cependant, en guise d'illustration, les exemples qu'il nous propose sont pris dans la synchronie, suggérant ainsi un système de transformations de structures correspondant à des différences d'attitude à l'égard des animaux, dans la zone subarctique, en Amérique et en Asie. On passe ainsi des Montagnais comme exemple typique d'animisme donateur aux Tchoukches de Sibérie chez lesquels sont associées des relations équilibrées avec l'esprit qui contrôle les rennes sauvages et des relations de dépendance vis-à-vis de celui qui protège les rennes domestiques. Avec les Bouriates des steppes mongoliennes, qui ont adopté l'élevage extensif des bovins et des chevaux, nous sommes dans un tout autre contexte culturel. Ici, plus de " collectifs égalitaires d'humains, d'esprits et d'animaux», mais, même s'il y subsiste "des poches d'identification animique », un collectif analogique qui "s'étend jusqu'aux limites du cosmos ».

Viennent ensuite des réflexions plus générales sur la domestication, en partant de l'idée que l'adoption de la domestication ne suffit pas à expliquer «la transition » qui vient d'être décrite. En effet, dit Descola, « la disponibilité d'un animal domesticable n'aboutit pas nécessairement à sa domestication » (p. 514). La preuve en est que les peuples de l'Amérique subarctique n'ont pas domestiqué le caribou, alors qu'ils l'ont fait du chien, et qu'ils n'ont même pas accepté des rennes domestiqués que l'administration a voulu leur imposer.

De même dans l'Amérique amazonienne, il n'y a eu aucune domestication alors que cela aurait été possible pour le tapir ou le pécari et que des camélidés ont été domestiqués dans l'Amérique andine. Pourtant en Amazonie, l'apprivoisement d'animaux de compagnie est fréquent, en particulier pour les petits dont les parents ont été tués à la chasse, mais ces animaux ne sont pas destinés à être mangés ni à se reproduire. Par ailleurs, dans les mêmes régions, des plantes ont été domestiquées très anciennement.

Certaines populations acceptant des animaux domestiques européens, comme le porc, ce ne serait pas la domestication en général que les Amérindiens amazoniens refuseraient, mais celle des animaux qu'ils chassent, en raison d'une impossibilité à transformer leur schème de 
relation à l'animal. «Il est plus facile d'adopter un objet technique nouveau que d'inventer une nouvelle relation technique» (p. 523). Descola réitère sa conviction que ce sont les modifications des rapports que les hommes entretiennent entre eux et avec le monde, et non pas le progrès technique en lui-même, qui entraînent les transformations.

\section{Quelle pertinence pour les principes sur lesquels sont construites les quatre ontologies?}

Les principes mêmes sur lesquels Descola construit ses quatre ontologies me paraissent critiquables. Tout d'abord, peut-on vraiment soutenir que l'identification est antérieure à la relation? Non seulement, il est obligé de multiplier les arguments pour nous en convaincre, mais ses propres données le contredisent et montrent que l'identification d'un existant prend en compte le contexte relationnel dans lequel il apparaît. Il en est ainsi de l'incident qu'il donne en exemple dès les premières pages, dans lequel la femme d'un chasseur achuar se fait mordre par un serpent : ce n'est pas la morsure elle-même qui est inquiétante, mais le fait inhabituel que ce serpent se soit aventuré très près des habitations. Il n'était donc pas un serpent ordinaire, mais la conséquence d'une faute commise par le chasseur qui avait tué beaucoup de singes laineux et en avait laissé agoniser sur place.

Cette importance du relationnel se confirme tout au long de l'ouvrage, et pas seulement dans la cinquième partie qui lui est consacrée, sans que l'on puisse vraiment, comme le voudrait Descola, distinguer un relationnel intrinsèque $\mathrm{d}$ 'un relationnel extrinsèque.

Ensuite, est-il vraiment nécessaire de faire une distinction si difficile à établir, comme il en convient lui-même à plusieurs reprises, entre intériorité et physicalité, alors que, pour deux de ses ontologies, l'une et l'autre sont, chez les humains et les non-humains, soit semblables, c'est-à-dire participant d'une même " essence » (le totémisme), soit différentes dans un jeu de correspondance (l'analogisme). Dans le naturalisme qui caractérise notre monde occidental, la frontière entre les deux ne cesse de se déplacer au gré des découvertes en neurosciences. Reste l'animisme où l'intériorité, au sens de Descola, semble tellement extensible qu'elle ne s'oppose plus qu'à l'apparence externe et au comportement.

En fait, comme la plupart des anthropologues, c'est à partir de son expérience de terrain que Descola essaye de comprendre les autres manières de penser le monde et les relations entre les différents existants. Quelle que soit la société étudiée, le plus difficile pour l'anthropologue est de rendre compte de ce qui est pour lui du domaine de la croyance et qui est réalité pour ses interlocuteurs. La seule façon de s'en tirer est de décrire toutes les pratiques dans lesquelles cette réalité se manifeste. Or, et c'est sans doute ce qui est le plus gênant dans cet ouvrage, on est le plus souvent plongé dans des discussions très abstraites.

Ainsi, il aborde la question du soi, du non-soi, de l'autre, du « je » et du sujet en philosophe. Pour ce qui concerne la pensée occidentale, il s'en tient à l'histoire des idées, c'est-à-dire à la pensée savante, sans faire état des pratiques populaires. Pour cela, il aurait fallu s'intéresser à des sociétés particulières et à leur fonctionnement concret.

\section{Compatibilités, incompatibilités dans le cadre des quatre ontologies}

Parmi les objectifs que Descola se propose d'atteindre, il en est qui me paraissent particulièrement intéressants à revisiter : repérer les compatibilités et les incompatibilités et comprendre comment on peut passer $\mathrm{d}^{\prime}$ un schème de relations à un autre.

Commençons par les incompatibilités retenues par Descola. Ainsi, l'absence de relations aux ancêtres dans les terres animistes de l'Amazonie est contestée par certains chercheurs qui disent qu'elle ne concerne pas tous les groupes (Chaumeil, 1997). L'opposition possession/transe chamanique n'est pas non plus avérée partout où il y a animisme; en effet, l'esprit avec lequel le chamane communique peut venir l'habiter, par exemple chez les Yanomami. Enfin, les références à la polarité chaud/froid, qui, pour Descola, relèverait de l'ontologie analogique, se rencontrent chez les animistes de l'Amazonie vénézuélienne, comme il en convient lui-même dans une note. Le fait que cette opposition soit utilisée dans des raisonnements analogiques concernant uniquement la santé n'est pas une raison suffisante pour l'écarter, étant donné l'importance des rapports au corps et à l'interprétation que l'on tire des sensations corporelles dans tous les groupes humains et pas seulement pour ceux auxquels Descola attribue une ontologie analogique.

L'appartenance à ce qu'il appelle l'ontologie animique ne suffit donc pas à expliquer les incompatibilités qu'il a relevées, et ce sont plutôt vers les divers types de relation aux non-humains - qu'il a lui-même mises en évidence qu'il faudrait chercher des corrélations.

Par ailleurs, les travaux des cognitivistes ont confirmé ce que l'on sait depuis les Grecs, c'est-à-dire que le raisonnement analogique joue un rôle fondamental dans la pensée. D'ailleurs, Descola l'évoque à plusieurs reprises, mais sans s'y attarder. On ne peut pourtant pas présenter des données montrant que la majeure partie des sociétés humaines relève de l'ontologie analogique sans chercher une relation entre ces deux faits. De même, il paraît hasardeux de considérer, ainsi que le fait Descola, comme conséquence de l'ontologie analogique, l'existence de grands empires centralisés comme réponse à 
la nécessité d'unifier ce qui serait trop dispersé dans une multitude de correspondances. Ceci est à mettre en relation avec sa remarque sur le holisme selon Dumont, dont il conteste l'universalité dans toutes les sociétés non-modernes et qui ne concernerait que les ontologies analogiques, alors que la subordination des individus au groupe semble bien se retrouver partout en dehors des sociétés modernes.

Ceci pose aussi la question de la coexistence de plusieurs ontologies, ce que Descola lui-même admet à plusieurs reprises et qu'il faudrait, à mon avis, examiner en relation avec leur répartition et dans une perspective évolutionniste.

\section{Rejet d'une perspective dynamique}

Dès l'avant-propos, Descola déclare vouloir s'en tenir à une analyse des structures et «différer l'étude de leur évolution ». Or, il n'est pas sûr qu'une vision évolutionniste bien comprise ne lui permettrait pas d'arriver plus rapidement à ses fins, c'est-à-dire mettre en évidence les différents schèmes de relation aux autres existants et détecter les compatibilités et les incompatibilités.

Les anthropologues se sont longtemps méfiés de l'évolutionnisme en raison des méfaits qu'il a entraînés sur la vision que l'on avait de l'histoire de l'humanité, en recherchant dans des sociétés contemporaines des caractéristiques de l'humanité primitive. Il faut pourtant bien affronter ce que l'on répète à satiété : l'évolution culturelle a remplacé l'évolution biologique. Ceci devient d'autant plus nécessaire que l'on a maintenant des données de plus en plus fiables, d'une part sur l'histoire de l'homme et des sociétés, d'autre part sur ce qui le caractérise, par rapport à ses parents primates les plus proches, du point de vue de son fonctionnement mental. Descola fait à plusieurs reprises référence à ce dernier aspect, mais, à mon avis, il n'en tire pas toutes les conséquences. Pour comprendre ce que nous sommes, il faut se placer dans une perspective évolutionniste, évolution biologique quand il s'agit des caractéristiques qui nous ont été transmises grâce à la sélection naturelle, en particulier les capacités «altruistes » qui permettent au groupe de survivre et de se reproduire. Une fois l'espèce humaine installée et quand l'évolution culturelle prend la relève, ses caractéristiques se diversifient dans des directions variées. Peut-on parler d'une "sélection culturelle»? Probablement, et elle a joué en particulier sur les capacités d'adaptation aux aléas environnementaux, qu'ils soient écologiques ou sociaux. Mais on peut supposer que cette évolution culturelle ne peut être en contradiction trop flagrante avec les caractéristiques de l'espèce humaine. Par exemple, le développement de l'individualisme ne peut-il nous placer dans une situation trop éloignée des capacités «altruistes » de l'espèce et de respect de la solidarité de groupe qui a constitué un avantage adaptatif?
Sans doute faudrait-il inverser certaines des perspectives de Descola. Ainsi, la nécessité de la prédation sur les plantes et les animaux fait partie des conditions initiales d'existence des primates. Avec l'apparition de la réflexivité chez l'homme, ses rapports avec les autres existants indispensables à sa survie s'inscrivent dans des systèmes de pratiques et de représentations soumis à des dynamiques évolutives et pouvant prendre des directions différentes.

Le plus facile à traiter dans une perspective évolutionniste est le totémisme australien. En effet, les Aborigènes sont arrivés en Australie il y environ 60000 ans. Les préhistoriens supposent qu'il y eut deux à trois vagues de migration qui se sont interrompues il y a longtemps, quand l'Australie a été isolée par la remontée du niveau des mers. Les premières populations ont vécu sur les côtes et ne se sont aventurées que progressivement à l'intérieur de ce continent, et surtout dans les zones désertiques. Nous avons donc un cas d'évolution culturelle en circuit fermé à partir de quelques petits groupes qui ont dû successivement affronter des milieux différents, puis une progression démographique qui a sans doute modifié leurs rapports au territoire. D'ailleurs, on a la preuve que certaines modifications dans les règles de mariage, et en particulier l'adoption du système de sections, pouvant être associées à un passage à la patrilinéarité lié à la territorialité, ne sont apparues, dans certains groupes, que très récemment durant le $X X^{\mathrm{e}}$ siècle.

Le fait de se trouver sur le parcours de vagues de migration ou dans un cul-de-sac a évidemment des conséquences sur les changements culturels. Mais certains culs-de-sac qui ont accueilli plusieurs vagues migratoires de différentes origines ont pu être aussi des creusets de transformation. L'influence des apports extérieurs a eu une grande importance dans l'évolution des sociétés selon des modalités diverses pouvant aller de la perméabilité, dont traite, par exemple, Sperber (1996) dans son ouvrage La Contagion des idées, où il parle même «d'épidémiologie des croyances », à, parfois en même temps, une volonté de différenciation, le plus souvent à partir de signes visibles dans l'ornementation des corps, des vêtements ou des outils (voir, par exemple, Lemonnier [1987] sur les flèches en Nouvelle-Guinée).

La prise en compte, pour l'évolution des cultures, des modalités de déplacement des populations s'applique particulièrement à l'Amérique, qui a été peuplée, peut-être plus tardivement que l'Australie, à partir de la Sibérie. Les formes qu'y a prises le totémisme sont très différentes de ce que l'on trouve en Australie. L'animisme s'y est développé sous des formes diverses, principalement dans les zones forestières, que ce soit au Nord ou au Sud, mais cela n'a pas empêché l'existence de sociétés hiérarchisées, aussi bien chez les chasseurs-cueilleurs que chez les agriculteurs où se sont même développés des empires, avec les Aztèques et les Incas, dans le cadre 
de sociétés qui, pour Descola, se réfèrent à l'ontologie analogique.

\section{La domestication et les relations aux plantes et aux animaux}

L'apparition de l'agriculture est sans doute le signe le plus manifeste d'une modification des relations aux plantes et aux animaux que Descola aborde à plusieurs reprises, sans traiter complètement la question. En fait, il s'intéresse surtout aux modifications des relations liées à l'adoption de l'élevage. Or, pour parvenir à comprendre le phénomène du passage à l'agriculture, il faut distinguer son adoption, qui est le cas le plus répandu, de son invention qui n'a eu lieu qu'en certains points, mais, il faut le noter, à peu près à la même époque à l'échelle de l'évolution humaine, c'est-à-dire il y a environ 11000 à 6000 ans, les premières au Proche-Orient, en Asie du Sud-Est et en Nouvelle-Guinée.

Il faut aussi distinguer la domestication des plantes de celle des animaux. Que cette domestication implique un certain type de relation aux autres êtres vivants et qu'elle soit inhibée par d'autres, cela est certain, et Descola le montre bien dans le cas de la répugnance à domestiquer le gibier dans l'Amazonie animiste. Cependant, il n'explique pas pourquoi la même répugnance n'aurait pas joué vis-à-vis des plantes, notamment du manioc dont la domestication semble s'être produite dans cette même région. En particulier, il n'exploite pas la piste de réflexion que pourrait être, chez les Achuar, la différence d'attitude entre le gibier, chassé par les hommes et traité comme des affins, et le manioc, planté par les femmes et considéré comme leurs enfants, c'est-à-dire comme des êtres qu'il faut protéger, même si ce manioc peut se montrer agressif à l'égard de ces mêmes femmes et de leur descendance humaine. La distinction entre les animaux que l'on chasse et ceux que l'on élève se retrouve dans de nombreuses régions, y compris au Proche-Orient et en Europe.

Faut-il qu'un non-humain cesse d'être un alter ego pour pouvoir être domestiqué ? Il semble que le processus soit plus complexe, et les mythes d'origine souvent réactivés dans les rituels agraires sont autant de pistes à explorer.

Il est curieux qu'à propos du Proche-Orient, Descola fasse référence à une nécessité matérielle pour expliquer le passage à l'agriculture, alors que les données recueillies par les archéologues l'infirment. Les populations étaient sédentarisées depuis 2000 ans quand elles sont passées de la chasse-cueillette à l'agriculture, et le changement le plus visible qui a précédé ce passage est dans l'organisation spatiale des villages avec intégration des tombes et la spécialisation de l'alimentation sur certaines plantes et animaux, ceux-là mêmes qui allaient être domestiqués (Cauvin 1994). Tout cela indique donc bien une modification socioculturelle précédant l'apparition de l'agriculture, et non une diminution des ressources, modification dans laquelle les relations aux lieux ont certainement joué un rôle.

Descola évoque bien ces changements corrélés entre techniques, modes de vie et attitudes vis-à-vis de l'audelà, mais c'est au début de l'ouvrage, dans le chapitre «Le sauvage et le domestique », où il évoque la comparaison que fait Dwyer (1996) entre trois populations $\mathrm{d}$ 'horticulteurs de Nouvelle-Guinée en fonction du degré d'anthropisation de leur milieu. Les premiers, peu nombreux, font peu de différence entre la zone habitée et la forêt, dormant aussi bien dans l'une que dans l'autre. Les esprits, et notamment l'âme des morts, les accompagnent partout. Chez les seconds, qui ont une agriculture plus développée et une densité beaucoup plus forte, l'âme des défunts finit, après plusieurs étapes, par s'installer aux confins de leur territoire. Les troisièmes, enfin, fortement sédentarisés, pratiquent une agriculture intensive avec élevage de porcs et fréquentent peu les reliquats de forêts dans les montagnes; les esprits y sont relégués dans des lieux inaccessibles et ne communiquent avec les humains que par l'intermédiaire d'oiseaux ou d'objets rituels. On aurait aimé qu'en fin de parcours, Descola revienne sur cet exemple et nous dise de quel type d'ontologie ces populations relèvent à son avis.

C'est un des autres problèmes de cet ouvrage : donner trop souvent l'impression de ne fournir que des exemples choisis pour illustrer une thèse, alors que les contreexemples foisonnent. C'est le cas, en particulier, pour toutes les sociétés qui semblent par défaut relever de ce que Descola appelle l'ontologie analogique, mais dans lesquelles on pressent déceler de l'animisme et parfois même du totémisme. Le problème est que les données recueillies par les anthropologues sur d'autres thèmes de recherche ne nous éclairent pas toujours dans ce domaine. Je prendrai donc l'exemple des Bunaq de Timor sur lesquels j'ai moi-même travaillé. Chez eux, les relations aux ancêtres sont très importantes. On doit les nourrir et leur donner du bétel chaque fois qu'on les convoque pour leur demander leur aide. On y trouve aussi des êtres de l'au-delà maîtres des plantes, par exemple pour celles qui entrent dans la construction des maisons; il faut les remercier par des offrandes quand on les utilise. Les correspondances analogiques jouent un rôle important pour la compréhension des techniques thérapeutiques, mais aussi dans l'explicitation des mythes étiologiques. Tous les êtres vivants ont une âme, les humains en ayant deux, l'une appelée «âme chair», et l'autre, "âme qui vole». Le riz a un statut ambigu, à la fois comme métamorphose des sangliers, eux-mêmes émanations des maîtres du sol, et comme métamorphose d'un être humain. Des interdits d'utilisation ou de consommation de certaines plantes et animaux frappant certaines maisons lignagères sont considérés de «descendance » et s'apparentent ainsi 
à des relations de type totémique. Il en est de même de la conviction d'un chef de village concernant les liens l'unissant à un serpent vivant dans le banian situé à la principale source d'eau potable de ce village. À quelle type d'ontologie Descola rattacherait-il cette population?

Que dire de nos propres relations aux plantes et aux animaux? L'opposition nature/culture n'empêche nullement certains de prêter, dans certaines circonstances, une intentionnalité à certains animaux ou même à des plantes. Le vertige analogique ne nous épargne pas, comme dans toute culture où l'écriture permet une démultiplication des possibilités de correspondances entre les éléments de l'univers.

\section{Conclusion}

À mettre au bénéfice de cet ouvrage, il y a tout d'abord l'exploration des différentes facettes que prennent, dans d'autres cultures que la nôtre, les diverses façons de considérer les non-humains comme des partenaires à part entière, doués d'intentionnalité, dont les actions et les réactions interviennent dans la vie des humains. Ce faisant, il détruit les images trop simplistes qui se sont répandues dans le grand public sur les relations que soit les chasseurs-cueilleurs, soit les Amérindiens entretiennent avec les plantes et les animaux qui peuplent leur environnement, en montrant à quel point elles peuvent être diverses.

À travers ce livre, nous voyons que, si pour de nombreux peuples l'opposition nature/culture n'a pas de sens, par contre, la présence de l'invisible dans la vie quotidienne occupe une place importante parce que cet invisible n'est pas le fait de divinités lointaines et encore moins d'un Dieu unique et impersonnel, mais qu'il est ancré dans l'espace de vie ou qu'il appartient à celui dans lequel on se projette, et dans chacun des existants qui l'animent. Cependant, les relations au territoire et à ses «lieux» particuliers, aspect important des relations qu'une société entretient avec son espace de vie, sont peu développées par Descola.

Notons enfin qu'il est un aspect omniprésent dont Descola ne nous montre pas assez les différentes formes qu'il peut prendre : c'est le sentiment d'avoir une dette à l'égard de ces êtres demeurant le plus souvent par-delà le visible, mais qui sont maîtres de tout ce dont dépend la vie de chacun.

Le livre de Descola constitue néanmoins une étape importante dans nos réflexions face au foisonnement des données recueillies par les anthropologues et aux découvertes des sciences cognitives et des éthologues. Mais nous permet-il vraiment de nous situer dans l'histoire des relations que les sociétés humaines ont entretenues avec les autres êtres qui peuplent leur environnement? Devant les attitudes diverses développées par nos contemporains habités par le sentiment confus qu'ils dépendent de l'existence de ces êtres autant que ceux-ci dépendent de leurs actions, il est urgent de continuer à investir le champ de recherches que nous offre cet ouvrage.

\section{Références}

Cauvin, J., 1994. Naissance des divinités, naissance de l'agriculture: la révolution des symboles au néolithique, Paris, CNRS Éditions.

Chaumeil, J.-P., 1997. Les os, les flûtes, les morts. Mémoire et traitement funéraire en Amazonie, Journal de la Société des américanistes, 83, 83-110.

Dwyer, P.D., 1996. The invention of nature, in Ellen, R., Fukui, K. (Eds), Redefining Nature: Ecology, Culture, and Domestication, Oxford (UK)/Washington (DC), Berg, 157-186.

Lemonnier, P., 1987. Le sens des flèches. Culture matérielle et identité ethnique chez les Anga de Nouvelle-Guinée, in Koechlin, B., Sigaut, F., Thomas, J.M.C., Toffin, G., De la voûte céleste au terroir, du jardin au foyer : mosaïque sociographique, texte offerts à Lucien Bernot, Paris, EHESS.

Sperber, D., 1996. La Contagion des idées : théorie naturaliste de la culture, Paris, Odile Jacob. 